\title{
Levels of interleukin-6, superoxide dismutase and malondialdehyde in the lung tissue of a rat model of hypoxia-induced acute pulmonary edema
}

\author{
HENGBO GAO $^{1}$, YINGPING TIAN ${ }^{1}$, WEI WANG ${ }^{2}$, DONGQI YAO ${ }^{1}$, TUOKANG ZHENG $^{1}$ and QINGBING MENG $^{1}$ \\ ${ }^{1}$ Department of Emergency Medicine, The Second Hospital of Hebei Medical University, Shijiazhuang, Hebei 050000; \\ ${ }^{2}$ Department of Hepatology, The Fifth Hospital of Shijiazhuang, Shijiazhuang, Hebei 050021, P.R. China
}

Received November 4, 2014; Accepted November 30, 2015

DOI: $10.3892 /$ etm.2015.2962

\begin{abstract}
The present study aimed to investigate the levels of malondialdehyde (MDA), superoxide dismutase (SOD) and interleukin (IL)-6 in the lung tissue of a rat model of acute pulmonary edema induced by acute hypoxia, and its pathophysiological significance. A total of 48 adult Wistar rats were randomly divided into group $\mathrm{A}$, a normal group; group $\mathrm{B}$, a model of acute pulmonary edema induced by hypoxia for $24 \mathrm{~h}$; group C, a model of acute pulmonary edema induced by hypoxia for $48 \mathrm{~h}$; and group $\mathrm{D}$, a model of acute pulmonary edema induced by hypoxia for $72 \mathrm{~h}$. The rats in groups B-D were intraperitoneally injected with $6 \%$ ammonium chloride to establish the model of acute pulmonary edema, and were subsequently sacrificed following successful modeling for 24, 48 and $72 \mathrm{~h}$. The plasma of rats was isolated and the lungs of the rats were removed. Subsequently, a 10\% lung homogenate was prepared and the contents and the activities of MDA, SOD and IL-6 in the lung tissue and IL-6 in the plasma were detected by enzyme-linked immunosorbent assay. MDA and IL-6 expression levels increased and SOD activity decreased in the lung tissue in group B as compared with group A; however the difference did not reach significance $(\mathrm{P}>0.05)$. MDA, IL-6 and SOD levels in the lung tissue of rats were significantly altered following the increased duration of pulmonary edema in groups $\mathrm{C}$ and $\mathrm{D}$, as compared group $\mathrm{A}(\mathrm{P}<0.05)$. The plasma IL-6 levels of the rats in groups B-D significantly increased, as compared with those in group $\mathrm{A}(\mathrm{P}<0.05)$. In conclusion, the results of the present study demonstrated that the incidence of acute pulmonary edema may be associated with oxidative stress. Furthermore, decreased antioxidant capacity and increased free radical levels may be associated with pulmonary edema, as in the present study the levels of IL-6, SOD
\end{abstract}

Correspondence to: Dr Yingping Tian, Department of Emergency Medicine, The Second Hospital of Hebei Medical University, 215 Heping West Road, Shijiazhuang, Hebei 050000, P.R. China E-mail: yingpingtian@126.com

Key words: acute pulmonary edema, malondialdehyde, superoxide dismutase, interleukin-6 and MDA in the lung tissue were observed to be associated with the pathological changes of the disease.

\section{Introduction}

Acute pulmonary edema is a common presentation in cardiology. Its clinical manifestations include sudden severe dyspnea and orthopnea, which are often accompanied by a cough, pink frothy sputum, restlessness, cyanosis, sweating, tachycardia, and moist and wheezing rales (1). If severe, acute pulmonary edema can cause syncope and cardiac arrest (2).

Acute pulmonary edema is pernicious and the mortality rate is $\sim 12 \%$ (3). The pathogenesis and physiology of acute pulmonary edema have been extensively researched in recent years $(2,4)$; however, the pathogenesis of acute pulmonary edema is yet to be fully elucidated. Previous studies have demonstrated that the following are important mechanisms of acute pulmonary edema: Excessive shrinkage of pulmonary vasculature; increased pulmonary capillary permeability; and a decreased capacity for the clearance of hypoxia-induced alveolar fluid $(5,6)$. The study of pathological and physiological changes of lung tissue in the human body is challenging. Therefore, the human pathologic state and pathophysiological processes may be reproduced in an animal model of lung tissue damage to enable investigation of the occurrence, development and characteristics of the disease, and to explore novel therapeutic strategies for the treatment of acute pulmonary edema.

Animal models of acute pulmonary edema have previously been established and studies have demonstrated that changes in the levels of malondialdehyde (MDA), superoxide dismutase (SOD) and interleukin (IL)-6 may play an important role in hypoxia-induced acute pulmonary edema $(7,8)$. In the present study, a rat model of acute pulmonary edema was established in order to clarify the functions of MDA, SOD and IL-6 in this condition. In particular, alterations in the levels of SOD and MDA in the lung tissue of rats with acute pulmonary edema were analyzed to elucidate the role of hypoxia in acute pulmonary edema.

\section{Materials and methods}

Experimental animals and grouping. A total of 48 adult Wistar rats (male, 26; female, 22; weight, 200 $\pm 20 \mathrm{~g}$ ) were purchased 
from the Experimental Animal Center of Zhengzhou University (Zhengzhou, China). The rats were divided into four equally sized groups: Group A, normal group; group B, acute pulmonary edema model induced by hypoxia for $24 \mathrm{~h}$; group $\mathrm{C}$, acute pulmonary edema model induced by hypoxia for $48 \mathrm{~h}$; and group $\mathrm{D}$, acute pulmonary edema model induced by hypoxia for $72 \mathrm{~h}$. The rat model of pulmonary edema was prepared in groups B-D by the injection of $6 \%$ ammonium chloride (as described below). The general condition of the rats was observed in addition to lung coefficient calculations and pathological sections, and the successful establishment of the model was verified. The present study was performed in strict accordance with the recommendations outlined by the Guide for the Care and Use of Laboratory Animals (8th edition, 2010) of the National Institutes of Health (Bethesda, MD, USA). The animal protocol was reviewed and approved by the Institutional Animal Care and Use Committee of the Second Hospital of Hebei Medical University (Shijiazhuang, China).

Establishment of the animal model and experimental procedures. In order to establish a rat model of acute pulmonary edema, the 36 rats in groups B-D were injected with 6\% ammonium chloride (Bohu Biotechnology Co., Shanghai, China) into the abdominal cavity. Female rats were administered $6-8 \mathrm{ml} / \mathrm{kg}$, whereas male rats received $9-12 \mathrm{ml} / \mathrm{kg}$. The animal model was established according to a previously described procedure (4). After the model was deemed successful, all rats were given ad libitum access to feed and water. Rats in the experimental groups were fasted $24 \mathrm{~h}$ prior to sampling, whereas rats in the normal group were fed during this time. Following successful modeling for 24, 48 and $72 \mathrm{~h}$, rats in groups B-D were anesthetized by the intraperitoneal injection of 10\% urethane (Nengren Biotechnology Co., Wuhan, China). Heparinized blood was harvested from the abdominal aorta and centrifuged at $4,500 \times \mathrm{g}$ for $15 \mathrm{~min}$ at $4^{\circ} \mathrm{C}$ to separate the plasma, which was subsequently preserved at $-70^{\circ} \mathrm{C}$ prior to determination of IL-6 levels. The rat lungs were separated and harvested in order to prepare the $10 \%$ lung tissue homogenate using the tissue lysate (Boster Biological Technology, Ltd., Wuhan, China). Hematoxylin and eosin staining was performed in strict accordance with the manufacturer's protocol (Dingguo Biotechnology Co. Ltd., Beijing, China). The main procedure included the following steps: Sampling and fixation, dehydration, paraffin embedding, slicing and wax dyeing. The staining results were observed under a light microscope (BX51; Olympus Corp., Tokyo, Japan).

Determination of water content in lung tissue. The surface blood in the left lung of the rats was dried using an absorbent strip, immediately weighed, and placed into a $60^{\circ} \mathrm{C}$ oven for $72 \mathrm{~h}$, until no further weight reduction was observed. Subsequently, the dry weight of the lung tissue was calculated and the water content (\%) in the lung tissue was determined as (wet weight - dry weight) / wet weight $\times 100 \%$.

Determination of MDA and SOD levels in lung tissue. MDA and SOD levels were detected in the lung tissue of rats in the four groups. MDA and SOD assay kits were purchased from Boster Biological Technology, Ltd., and were performed in strict accordance with the manufacturer's protocol.

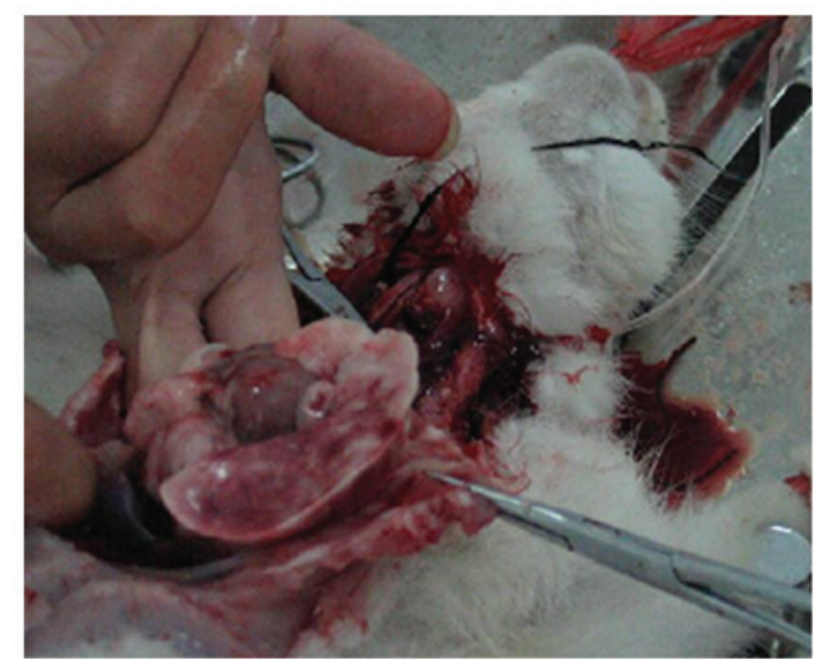

Figure 1. Significant edema was detected in the lung tissue of the rat models of acute pulmonary edema following sacrifice, and the wet weight was significantly increased.

Enzyme-linked immunosorbent assay (ELISA). IL-6 levels in the plasma and lung tissue were detected using ELISA (Sigma-Aldrich, St. Louis, MO, USA), according to the manufacturer's protocol.

Statistical analysis. Data were analyzed using SPSS statistical software, version 14.0 (SPSS, Inc., Chicago, IL, USA) and were presented as the mean \pm standard deviation. Between-group differences were compared using single factor analysis. $\mathrm{P}<0.05$ was considered to indicate a statistically significant difference.

\section{Results}

Observation results in the lung tissue of rats. Rats in the four groups were sacrificed, and lung tissue was obtained. In group A, the lung tissue was found to be uniform, with no significant pulmonary edema, and the wet weight was not significantly increased. However, significant edema was detected in the lung tissue of groups B, C and D, while the wet weight and water content were significantly increased ( $\mathrm{P}=0.046,0.021$ and 0.009 , respectively; Fig. 1) compared with group A. The results of hematoxylin and eosin staining showed that the rat lung tissue in group A did not present significant edema, and the pulmonary tissue morphology was normal. By contrast, pulmonary congestion and edema were detected in groups $\mathrm{B}, \mathrm{C}$ and $\mathrm{D}$, with a protein rich liquid saturating the pulmonary interstitium, alveoli and bronchioles, in addition to hyaline membranes lining the alveolar walls (Fig. 2).

MDA and SOD changes in the lung tissue of rats. The levels of MDA and SOD in the lung tissue of rats with acute hypoxia in groups B-D were measured as representative values in edema at different time points, and compared with those of group A. The results demonstrated that the MDA levels increased whereas SOD activity decreased in the rats in group B, as compared with those in group A, although not significantly $(\mathrm{P}>0.05)$. However, the MDA levels and SOD activity in the lung tissue of rats with pulmonary edema were significantly altered as the duration of edema increased. The MDA levels 
Table I. Superoxide dismutase (SOD) activity and malondialdehyde (MDA) levels in the lung tissue of rats in groups A-D.

\begin{tabular}{lllll}
\hline Indices & Group A & Group B & Group C & Group D \\
\hline SOD (U/g) & $3.57 \pm 0.67$ & $3.33 \pm 0.56$ & $2.74 \pm 0.54^{\mathrm{a}}$ & $2.56 \pm 0.48^{\mathrm{a}}$ \\
MDA (mmol/g) & $0.97 \pm 0.47$ & $1.12 \pm 0.56$ & $1.37 \pm 0.62^{\mathrm{a}}$ & $1.52 \pm 0.74^{\mathrm{a}}$ \\
\hline
\end{tabular}

Group A, normal group; group B, acute pulmonary edema model induced by hypoxia for $24 \mathrm{~h}$; group C, acute pulmonary edema model induced by hypoxia for $48 \mathrm{~h}$; and group $\mathrm{D}$, acute pulmonary edema model induced by hypoxia for $72 \mathrm{~h}$. ${ }^{\mathrm{a}} \mathrm{P}<0.05$, as compared with group A.

Table II. Levels of IL-6 in the plasma and lung tissue of rats in groups A-D.

\begin{tabular}{lrccc}
\hline Indices & Group A & Group B & Group C & Group D \\
\hline IL-6 in plasma $(\mu \mathrm{g} / \mathrm{l})$ & $103.83 \pm 32.14$ & $157.48 \pm 39.59^{\mathrm{a}}$ & $178.53 \pm 45.78^{\mathrm{a}, \mathrm{b}}$ & $194.91 \pm 54.59^{\mathrm{a}, \mathrm{b}, \mathrm{c}}$ \\
IL-6 in lung tissue $(\mu \mathrm{g} / \mathrm{l})$ & $97.58 \pm 31.67$ & $117.79 \pm 35.54$ & $146.60 \pm 43.81^{\mathrm{d}, \mathrm{e}}$ & $162.80 \pm 46.01^{\mathrm{d}, \mathrm{e}, \mathrm{f}}$ \\
\hline
\end{tabular}

Levels of IL-6 in plasma are significantly increased in the rats in groups B-D, as compared with group A; whereas levels of IL-6 in the lung tissue are significantly increased in the rats in groups $\mathrm{C}$ and $\mathrm{D}$, as compared with group A. Group A, normal group; group B, acute pulmonary edema model induced by hypoxia for $24 \mathrm{~h}$; group $\mathrm{C}$, acute pulmonary edema model induced by hypoxia for $48 \mathrm{~h}$; and group D, acute pulmonary edema model induced by hypoxia for $72 \mathrm{~h}$. ${ }^{\mathrm{a}} \mathrm{P}<0.05$, vs. group A plasma; ${ }^{\mathrm{b}} \mathrm{P}<0.05$, vs. group $\mathrm{B}$ plasma; ${ }^{\mathrm{P}}<0.05$, vs. group $\mathrm{C}$ plasma; ${ }^{\mathrm{d}} \mathrm{P}<0.05$, vs. group A lung tissue; ${ }^{\mathrm{P}}<0.05$, vs. group $\mathrm{B}$ lung tissue; ${ }^{\mathrm{f}} \mathrm{P}<0.05$, vs. group $\mathrm{C}$ lung tissue.
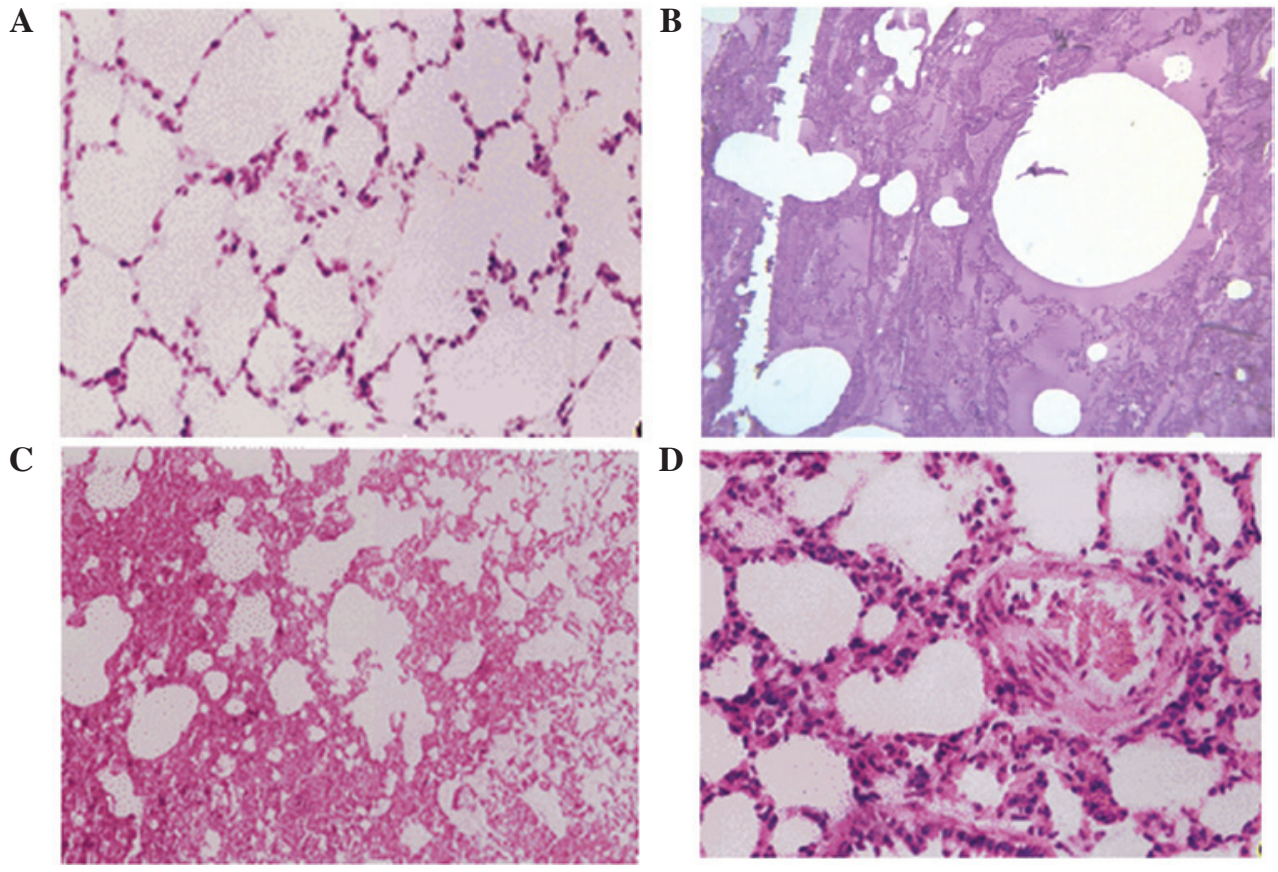

Figure 2. Hematoxylin and eosin stained sections of lung tissue harvested from various groups. (A) Group A: Normal pulmonary tissue morphology and no pulmonary edema detected (magnification, x100). (B) Group B (magnification, x40) and (C) group C (magnification, x10): Pulmonary congestion and edema were detected, with a protein rich liquid saturating the pulmonary interstitium, alveoli and bronchioles. (D) Group D: Pulmonary interstitial edema and thickening were detected, as well as inflammatory cell infiltration, while the alveolar cavity was filled with exudation (magnification, x20).

significantly increased in groups $\mathrm{C}$ and $\mathrm{D}$ following hypoxia for $48 \mathrm{~h}$ and $72 \mathrm{~h}$ whereas SOD activity significantly decreased, when compared with those in group $\mathrm{A}(\mathrm{P}<0.05$; Table I).

IL- 6 changes in the plasma and lung tissue of rats. The changes of IL-6 levels in the lung tissue and plasma of the rats with pulmonary edema induced by acute hypoxia were measured at three different time points, and compared with those in group A. The results showed that the IL-6 levels were significantly increased in the plasma of the rats in groups B-D as compared with those in group $A(P<0.05)$. The IL-6 levels in the lung tissue were notably increased in the rats in group B compared with those in group $\mathrm{A}(\mathrm{P}>0.05)$, and were significantly increased in the rats in groups $\mathrm{C}$ and $\mathrm{D}$ as compared with those in group $\mathrm{A}(\mathrm{P}<0.05)$, indicating that the IL- 6 levels increased when the duration of lung edema was prolonged (Table II). 


\section{Discussion}

Acute pulmonary edema predominantly occurs following acute extensive myocardial infarction, acute myocarditis, severe hypertension, aortic or mitral stenosis, infective endocarditis and cardiac trauma (9). Its onset is rapid, often inducing cardiac and respiratory failure; therefore, the mortality rates are high (10). In recent years, the incidence of acute pulmonary edema has gradually increased (3). Prevention and treatment can significantly increase the survival rate of patients with pulmonary edema and reduce the sequelae and complications (9). Since this disease has important social significance, it has been increasingly investigated by the medical community $(11,12)$.

Study of the pathological and physiological changes of lung tissue in the human body is difficult. Therefore, it is useful to reproduce the pathological state and pathophysiological processes of acute pulmonary edema in humans by stimulating lung tissue damage in rats. The creation of an animal model of acute pulmonary edema aids the elucidation of the occurrence, development and characteristics of the disease, and the exploration of novel therapeutic strategies for the treatment of acute pulmonary edema. Previous studies have reported the establishment of animal models of acute pulmonary edema $(7,8)$. It has previously been demonstrated that the following are key mechanisms of acute pulmonary edema: Excessive shrinkage of the pulmonary vasculature; increased pulmonary capillary permeability and a decreased capacity for the clearance of alveolar liquid induced by hypoxia (5). Previous studies have demonstrated that acute hypoxia induces oxidative stress; the latter is capable of regulating inflammation, reducing the synthesis and release of NO in lung tissue and reducing pulmonary $\mathrm{Na}^{+}-\mathrm{K}^{+} \mathrm{ATP}$ enzyme activity $(13,14)$. Therefore, oxidative stress induced by hypoxia may have a key role in the occurrence and development of acute pulmonary edema. In order to verify this hypothesis, the present study investigated the effects of oxidative stress in acute pulmonary edema by creating a rat model of hypoxia-induced acute pulmonary edema (8) and analyzing MDA, SOD and IL-6 levels.

Hypoxia can alter the balance of oxygen free radicals and nitric oxide. Oxygen free radicals are capable of inducing cell damage via the peroxidation of polyunsaturated fatty acids in the biofilm and peroxide decomposition products (15). The content or activity of important indicators of oxidative stress, including SOD, lipid peroxidation products such as MDA, and IL-6 can reflect the degree of lipid peroxidation and the extent of cell damage (16).

SOD is an important antioxidant enzyme that has a specific physiological activity and is the most important substance for scavenging free radicals in the body; furthermore, a decline in SOD levels is associated with aging. SOD is capable of resisting and blocking the damaging effects of oxygen free radicals on cells and initiating the timely repair of damage to cells caused by free radicals (17). Oxidative stress results from the excessive production of free radicals in the body, which can affect protein expression and other regulatory and response mechanisms (18). When induced by free radical and compensated stress, the antioxidant capacity of a cell or organism can increase, during which the phenomenon of transiently increased levels of SOD may be observed (19). SOD levels in the lung tissues of patients with acute pulmonary edema will increase due to oxidative stress. In particular, IL-6 is produced by various cells, including macrophages, $\mathrm{T}$ cells and $\mathrm{B}$ cells, and can regulate the growth and differentiation of numerous cells. Disorders in the expression levels of IL-6 have been associated with various diseases; for example, polyclonal B cell activation may occur in patients with autoimmune diseases, including systemic lupus erythematosus, Reiter syndrome, Castleman disease, scleroderma, membranoproliferative glomerulonephritis and rheumatoid arthritis (20). IL-6 expression levels are significantly increased in patients with these diseases, which may have an association with the patient's state of illness and curative effects. Furthermore, IL-6 expression levels are significantly increased in the lung tissue and plasma of patients with acute pulmonary edema, due to inflammation and oxidative stress. In particular, the severity of the patient's state of illness and prognosis increases as the IL-6 expression level increases (21).

The combined observation and detection of SOD, MDA, and IL-6 in the lung tissue of patients with acute pulmonary edema can comprehensively reflect alterations in the physiological state of patients, and is conducive to the effective diagnosis, treatment and prognosis of patients with acute pulmonary edema (22). In the present study, changes in the levels of MDA and SOD in the lung tissue of a rat model of hypoxia-induced acute pulmonary edema were compared at various durations of acute hypoxia and against the group A control. The results demonstrated that the levels of MDA increased in the lung tissue of the rats following the induction of acute pulmonary edema for $24 \mathrm{~h}$ whereas the SOD levels decreased at this time point $(\mathrm{P}>0.05)$. As the duration of pulmonary edema increased from $24 \mathrm{~h}$ in group $\mathrm{B}$ to 48 and $72 \mathrm{~h}$ in groups $\mathrm{C}$ and $\mathrm{D}$, the MDA and SOD levels in the lung tissue of the rats following hypoxia were significantly increased, as compared with those of the rats in group $\mathrm{A}(\mathrm{P}<0.05)$.

IL-6 levels in the lung tissue and plasma of rats in the three groups with various durations of induced acute hypoxia were compared with those in group A. The results demonstrated that plasma IL-6 levels were significantly increased in groups B-D, as compared with those in group $\mathrm{A}(\mathrm{P}<0.05)$. IL-6 levels in the lung tissue of the rats in group B notably increased following $24 \mathrm{~h}$ acute pulmonary edema, as compared with those in group A ( $>>0.05)$. With the increased duration of pulmonary edema, the IL-6 levels in the lung tissue of the rats in groups $C$ and $\mathrm{D}$ were significantly increased as compared with those in group $\mathrm{A}(\mathrm{P}<0.05)$. The results of the present study were consistent with the initial hypothesis.

Hypoxia-induced alterations in the oxidative stress indices of rats with acute pulmonary edema were observed in the present study, and the effects on the activity level of the antioxidant enzyme SOD, and the levels of the lipid peroxidation product MDA and IL-6 in were investigated the lung tissue, so as to explore their effects and related mechanisms in a rat model of acute pulmonary edema $(23,24)$. The results of the present study demonstrated that the antioxidant capacity and associated SOD enzyme activity of the lung tissue significantly decreased when the duration of pulmonary edema was extended; whereas the levels of the MDA lipid peroxidation product significantly increased, and the levels of IL-6 in the plasma and lung tissue significantly increased, which 
suggested that the induction of oxidative stress may have an important role in the pulmonary tissue damage associated with acute pulmonary edema. Furthermore, the alterations in the levels of the MDA, SOD and IL-6 oxidative stress-related indices in the lung tissue of rats with hypoxia suggested that the pathogenesis of acute pulmonary edema is complicated, and may be a result numerous factors $(25,26)$.

MDA, SOD and IL-6 were detected in the present study, and the results demonstrated that oxidative stress may have an important role in the occurrence of acute pulmonary edema. The present study had certain limitations. The number of rats used to model acute pulmonary edema was small and, at only 72 days, the observation time was relatively short. Furthermore, the present study was not a randomized prospective double-blind study. These limitations will be improved in further research. Therefore, definitive conclusions cannot be drawn from the results of the present study. Future studies of acute pulmonary edema with longer follow-up times and a larger sample size are required. However, the findings of the present study suggest that the simultaneous measurement of MDA, SOD and IL-6 levels in the lung tissue and plasma of rats with acute pulmonary edema may provide key guidance for the assessment of disease pathology, therapeutic strategies and prognosis for patients with acute pulmonary edema.

\section{References}

1. Obiagwu C, Paul V, Chadha S, Hollander G and Shani J: Acute pulmonary edema secondary to hyperbaric oxygen therapy. Oxf Med Case Reports 2015: 183-184, 2015.

2. Smith WS and Matthay MA: Evidence for a hydrostatic mechanism in human neurogenic pulmonary edema. Chest 111 : $1326-1333,1997$.

3. Wang XT, Liu DW, Zhang HM and Chai WZ: Integrated cardiopulmonary sonography: a useful tool for assessment of acute pulmonary edema in the intensive care unit. J Ultrasound Med 33: 1231-1239, 2014

4. MacIver DH and Clark AL: The vital role of the right ventricle in the pathogenesis of acute pulmonary edema. Am J Cardiol 115: 992-1000, 2015.

5. Theodore J and Robin ED: Pathogenesis of neurogenic pulmonary oedema. Lancet 2: 749-751, 1975.

6. Mammoto T, Jiang E, Jiang A, Lu Y, Juan AM, Chen J and Mammoto A: Twist 1 controls lung vascular permeability and endotoxin-induced pulmonary edema by altering Tie 2 expression. PLoS One 8: e73407, 2013.

7. Minnear FL, Kite C, Hill LA and van der Zee H: Endothelial injury and pulmonary congestion characterize neurogenic pulmonary edema in rabbits. J Appl Physiol (1985) 63: 335-341, 1987.
8. Zhang XM, Sun DY, Tang L and Yuan YJ: Preliminary experimental research on glucocorticoid for treatment of nitrogen dioxide induced acute pulmonary edema in rats. Zhonghua Lao Dong Wei Sheng Zhi Ye Bing Za Zhi 28: 822-826, 2010 (In Chinese).

9. Viswanathan S, Muthu V and Remalayam B: Pulmonary edema in near hanging. J Trauma Acute Care Surg 72: 297-301, 2012.

10. Hamdy O, Maekawa H, Shimada Y, Feng GG and Ishikawa N: Role of central nervous system nitric oxide in the development of neurogenic pulmonary edema in rats. Crit Care Med 29: 1222-1228, 2001.

11. Lionte C, Sorodoc L and Laba V: Respiratory syndromes in acute poisoning. Rev Med Chir Soc Med Nat Iasi 108: 544-548, 2004 (In Romanian)

12. Ekman I, Ekstrand L and Schaufelberger M: Pulmonary oedema - a life threatening disease. Eur J Cardiovasc Nurs 6: 259-264, 2007.

13. Greenbaum R, Bay J, Hargreaves MD, Kain ML, Kelman GR, Nunn JF, Prys-Roberts C and Siebold K: Effects of higher oxides of nitrogen on the anaesthetized dog. Br J Anaesth 39: 393-404, 1967.

14. Lavie L: Oxidative stress in obstructive sleep apnea and intermittent hypoxia - revisited - the bad ugly and good: Implications to the heart and brain. Sleep Med Rev 20: 27-45, 2015.

15. Wojtczak L and Slyshenkov VS: Protection by pantothenic acid against apoptosis and cell damage by oxygen free radicals - the role of glutathione. Biofactors 17: 61-73, 2003.

16. Prys-Roberts C: Principles of treatment of poisoning by higher oxides of nitrogen. Br J Anaesth 39: 432-439, 1967.

17. Mansuroğlu B, Derman S, Yaba A and Kizilbey K: Protective effect of chemically modified SOD on lipid peroxidation and antioxidant status in diabetic rats. Int J Biol Macromol 72: 79-87, 2015.

18. Li K, Gao B, Li J, Chen H, Li Y, Wei Y, Gong D, Gao J, Zhang J, Tan W, et al: ZNF32 protects against oxidative stress-induced apoptosis by modulating C1QBP transcription. Oncotarget 6: 38107-38126, 2015

19. Parsons PE: Respiratory failure as a result of drugs, overdoses, and poisonings. Clin Chest Med 15: 93-102, 1994.

20. Fang LL and Ye JJ: Investigated progress of expression of interleukin-6,-10 in herniated disc. Yi Xue Zong Shu 13: 569-572, 2007 (In Chinese).

21. Rassler B: Role of $\alpha$ - and $\beta$-adrenergic mechanisms in the pathogenesis of pulmonary injuries characterized by edema, inflammation and fibrosis. Cardiovasc Hematol Disord Drug Targets 13: 197-207, 2013

22. Sarada S, Himadri P, Mishra C, Geetali P, Ram MS and Ilavazhagan G: Role of oxidative stress and NFkB in hypoxia-induced pulmonary edema. Exp Biol Med (Maywood) 233: 1088-1098, 2008.

23. Chatterjee K and Parmley WW: The role of vasodilator therapy in heart failure. Prog Cardiovasc Dis 19: 301-325, 1977.

24. Sonnenblick EH, Mancini DM and LeJemtel TH: New positive inotropic drugs for the treatment of congestive heart failure. Am J Cardiol 55: 41 A-44A, 1985.

25. Francois G, Faizende J and Reboul J: Pulmonary edemas due to acute heroin poisoning. Ann Anesthesiol Fr 16: 77-83, 1975 (In French).

26. Hamdy O, Maekawa H, Shimada Y, Feng GG and Ishikawa N: Role of central nervous system nitric oxide in the development of neurogenic pulmonary edema in rats. Crit Care Med 29: $1222-1228,2001$. 